\title{
An Automatic Design Optimization Tool and its Application to Computational Fluid Dynamics
}

\author{
David Abramson $\dagger$, Andrew Lewis $\S$, Tom Peachey $\dagger$, Clive Fletcher $\ddagger$ \\ $\dagger$ Computer Science \& Soft. Eng. \\ Monash University, \\ $\S$ HPC Facility \\ $\$$ CANCES, \\ CLAYTON, VIC 3800, \\ Griffith University, \\ Nathan, Qld, 4111, \\ University of New South Wales \\ Australia \\ Australia. \\ Australian Technology Park \\ Eveleigh NSW 1430 \\ Australia
}

\begin{abstract}
In this paper we describe the Nimrod/O design optimization tool, and its application in computational fluid dynamics. Nimrod/O facilitates the use of an arbitrary computational model to drive an automatic optimization process. This means that the user can parameterise an arbitrary problem, and then ask the tool to compute the parameter values that minimize or maximise a design objective function. The paper describes the Nimrod/O system, and then discusses a case study in the evaluation of an aerofoil problem. The problem involves computing the shape and angle of attack of the aerofoil that maximises the lift to drag ratio. The results show that our general approach is extremely flexible and delivers better results than a program that was developed specifically for the problem. Moreover, it only took us a few hours to set up the tool for the new problem and required no software development.
\end{abstract}

\section{Introduction}

Computational science and engineering techniques have allowed a major change in the way that products can be engineered. Rather than building real world prototypes and performing experiments, a user can build a computational model that simulates the physical processes. Using such a model, many design alternatives can be explored computationally in a fraction of the time required. The technique has been applied widely in the areas of aviation, automotive engineering, environmental assessment and electromagnetics.

In the past, we have produced a number of tools for assisting a user in performing a rigorous design experiment using an arbitrary computational model. The Nimrod [1] and EnFuzion [2] tools make it possible to describe a number of discrete design scenarios using a simple declarative programming language. The system then produces a number of discrete scenarios, each a unique combination of parameter values from the cross-product of the parameter ranges. If integer or floating point parameters are specified, a step count is used to discretise the domain. The scheme is very powerful, and has been used in a number of real world problems [3]. In order to speed the execution of the experiment, distributed computers are used seamlessly to explore multiple scenarios in parallel. Whilst Nimrod and EnFuzion are optimized for clusters of computers, a "Grid Aware" version of Nimrod, called Nimrod/G [18], utilises resources on a global computational grid [4].

The biggest disadvantage of Nimrod is that when very large search spaces are specified, or when a high degree of resolution in the parameter values is required, the number of scenarios may exceed the computational power available. Further, the user may not actually want to explore all design space, but may be satisfied with a "good" solution instead. This background motivated the development of Nimrod/O, a variant of the Nimrod system that performs a guided search of the design space, rather than exploring all combinations. Nimrod/O allows a user to phrase a question like: "What set of design parameters will minimise (or maxmise) the output of my model?". If the model computes metrics like cost, lifetime, etc, then it is possible to perform automatic optimal design.

We believe the Nimrod/O system is unique in its ability to solve aribtrary problems without requiring the user to develop optimization code. In general, there are very few systems that allow a user to embed a 
computational model within a larger problem solving environment, let alone perform automatic optimization. Some systems which have some of these properties are DAKOTA, NEOS, NetSolve and SciRun. The DAKOTA project at Sandia laboratories investigated the combination of optimization with computational models [5]. DAKOTA is a $\mathrm{C}++$ based tool kit that allows a user to choose different optimization algorithms to guide the search for optimal parameter settings. DAKOTA has been successfully demonstrated on a number of structural mechanics applications. However, DAKOTA did not support rapid prototyping and still requires the construction of new programs for each different type of experiment which is performed. NEOS [6] is a distributed web based optimization engine that allows a user to solve optimization problems using special remote optimization servers. However, NEOS relies on the objective function being specified in an algebraic form and does not support objective functions implemented by CS\&E models. Likewise, NetSolve [7] provides a web based engine for solving linear algebra problems, but does not explicitly address optimization using CS\&E objective functions. SciRun [8], and its follow on Uintah [9], are interactive tools that allow a user to build a CS\&E model very rapidly using a graphical programming interface. However, they do not support optimization In contrast, Nimrod/O supports optimization, distributed computing and rapid prototyping in one tool.

In this paper we will discuss the Nimrod/O system and its application to a real world case study, the design of a simple, but optimal, aerofoil. We begin with a general discussion of automatic design optimization and the challenges it poses, and then expose the Nimrod/O architecture. We compare the performance of Nimrod/O at solving the aerofoil design against a tailored solution built in Fortran.

\section{Searching for Optimal Designs}

Design optimization is not new. There are many examples, particularly from the operations research literature, of the use of optimization theory to find good solutions to real world problems [10]. However, almost all of this work has assumed that the objective function can be expressed algebraically. This means that it is possible to evaluate the function quickly when a new set of design parameters are generated. Further, it is often possible to differentiate the function, which assists algorithms that try to perform gradient descent.

We are concerned with designs that are so complex that their effectiveness can only be evaluated by running a computational model. An important design goal is that the exact nature of the model is not important, and may be solved by a discrete event simulation or the solution of a set of partial differential equations. Because of this generality, it is necessary to run the model from the beginning each time the parameters are changed. Further, if derivatives of the objective function are required these are typically calculated using a finite difference approximation [11]. Because of this, the cost of executing the optimization algorithm is almost totally dominated by the cost of running the computational model.

From a user's perspective, the problem can be phrased simply - minimise (or maximise) the objective function across a set of parameters. Because almost all real world problems have bounds on the legal parameter values, it is possible to bound the search by these limits. Further, it is often necessary to place further constraints on the solution. For example, additional functions that combine parameter values can be used to further reduce the domain. We allow a user to specify both hard and soft constraints. Hard constraints are enforced during the search process itself. If a hard constraint would be violated by a particular choice of parameter values, then that part of space is not explored. In contrast, soft constraints are implemented by adding a penalty value to the objective function. Accordingly, soft constraints can be violated during the search, but the objective function is artificially higher than if the constraint was satisfied. These techniques are standard in non-linear optimization [12].

In general, the objective functions under consideration are non-linear, may not be smooth and may contain a high degree of noise. In addition, parameters may be continuous or discrete, depending on the nature of the underlying problem. Thus, no single optimization procedure will work for all problems. Some problems will contain multiple local minima, and it is impossible to guarantee that any one search algorithm will find the global minimum. Accordingly, Nimrod/O supports a range of algorithms, which can be executed multiple times (in parallel) from different starting locations. When a number of algorithms are used, each with different starting locations, it is possible to gain some insight into the nature of the objective function, 
and to generate a number of potential solution to the problem. Regardless of the search technique that is used, we assume that the computational model is well formulated, stable and robust across the parameter ranges.

\section{Nimrod/O Search Algorithms (to be expanded in final paper)}

At present, we have implemented four optimisation algorithms, namely a gradient search code called PBFGS [13][12], a Simplex search [20], a Divide-and-Conquer heuristic [13] and Simulated Annealing [23]. In this section we give a brief introduction to each of these algorithms. More details can be found in [13][14]. This section will be expanded in the final paper. In the case study reported in the paper we only used two of these algorithms, P-BFGS and Simplex.

\section{Nimrod/O Architecture}

Figure 1 shows a schematic of the Nimrod/O architecture. Nimod/O accepts a superset of the declarative "plan" files that are used to drive Nimrod, as discussed in [13]. In Nimrod/O additional statements are included that describe the optimization process that is to be used. Figure 2 shows and example of such a plan file, highlighting some of the new statements in Nimrod/O.

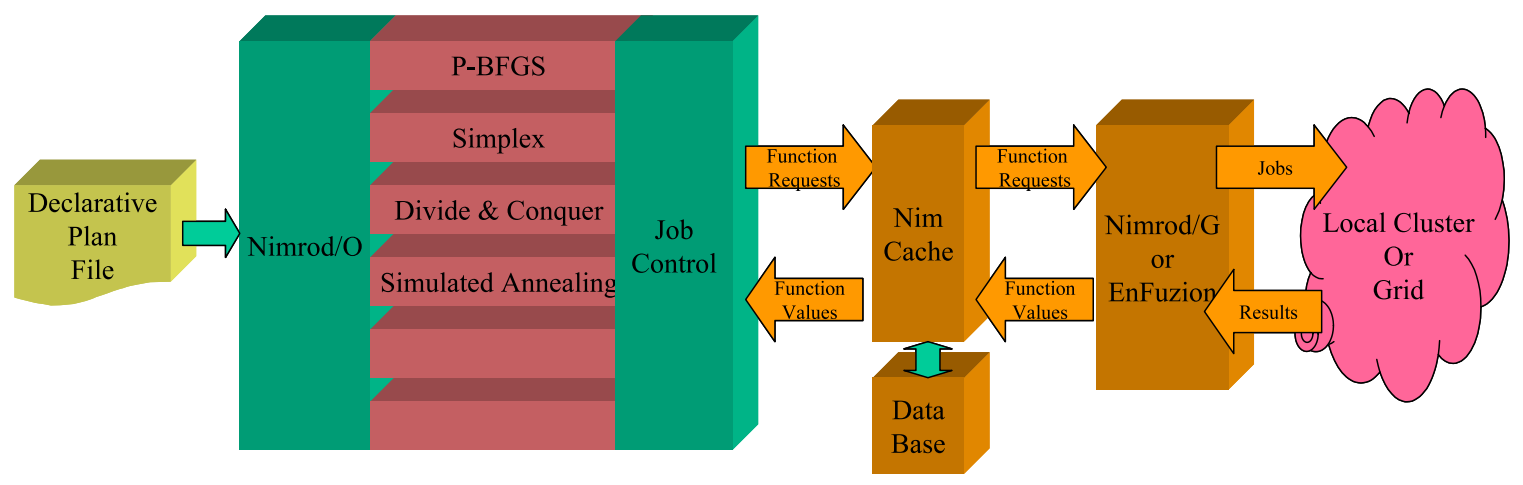

Figure 1 - Architecture of Nimrod/O

Nimrod/O has been built to support an extensible range of optimization procedures. Each of these procedures requires the evaluation of an objective function in order to proceed. This is performed by a request to the Nimrod/G or EnFuzion remote job execution engines. The algorithm forms a set of parameter values and passes these to Nimrod/G or EnFuzion for evaluation against the model. The model is run on an appropriate platform and the objective function value is extracted from the model output. A cache is superimposed between Nimrod/O and the backend to reduce the number of calculations required if the same parameter values are requested more then once. A persistent database is attached to the cache to support restart if Nimrod/O is terminated prematurely. By storing all function values, the user can restart the system from scratch and proceed to the same position without rerunning the computational models, providing a recovery process in the event of machine or network failure.

Nimrod/G and EnFuzion share a common API, and thus it is possible to execute the Nimrod/O model computations either on a local cluster, or on the Grid, depending on the available resources. This choice is transparent to the algorithms in Nimrod/O, and the selection of backend can be left to the user depending on the available resources and the number of processors required. Figure 3 shows the Nimrod/G and EnFuzion dispatchers in use. In the Nimrod screen on the left, the coloured boxes represent the different instances of the model, and these can be seen assigned to hosts on the Grid. In this particular example Nimrod is supporting three different Grid middleware services, namely, Legion[15], Globus [16] and a Condor [17]. The EnFuzion screen dump shows the different model instances as they run on the various nodes of the local cluster. The differences between Nimrod/G and EnFuzion are discussed in other papers [18]. 


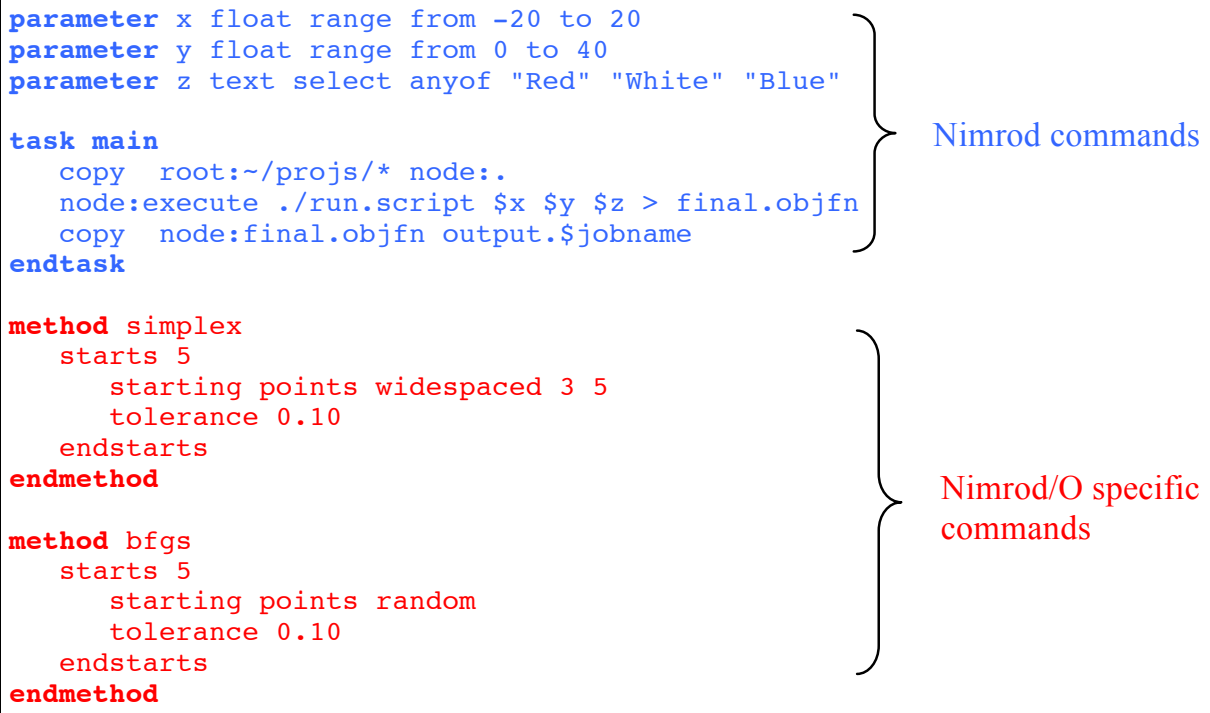

Figure 2 - A sample Nimrod/O plan file.

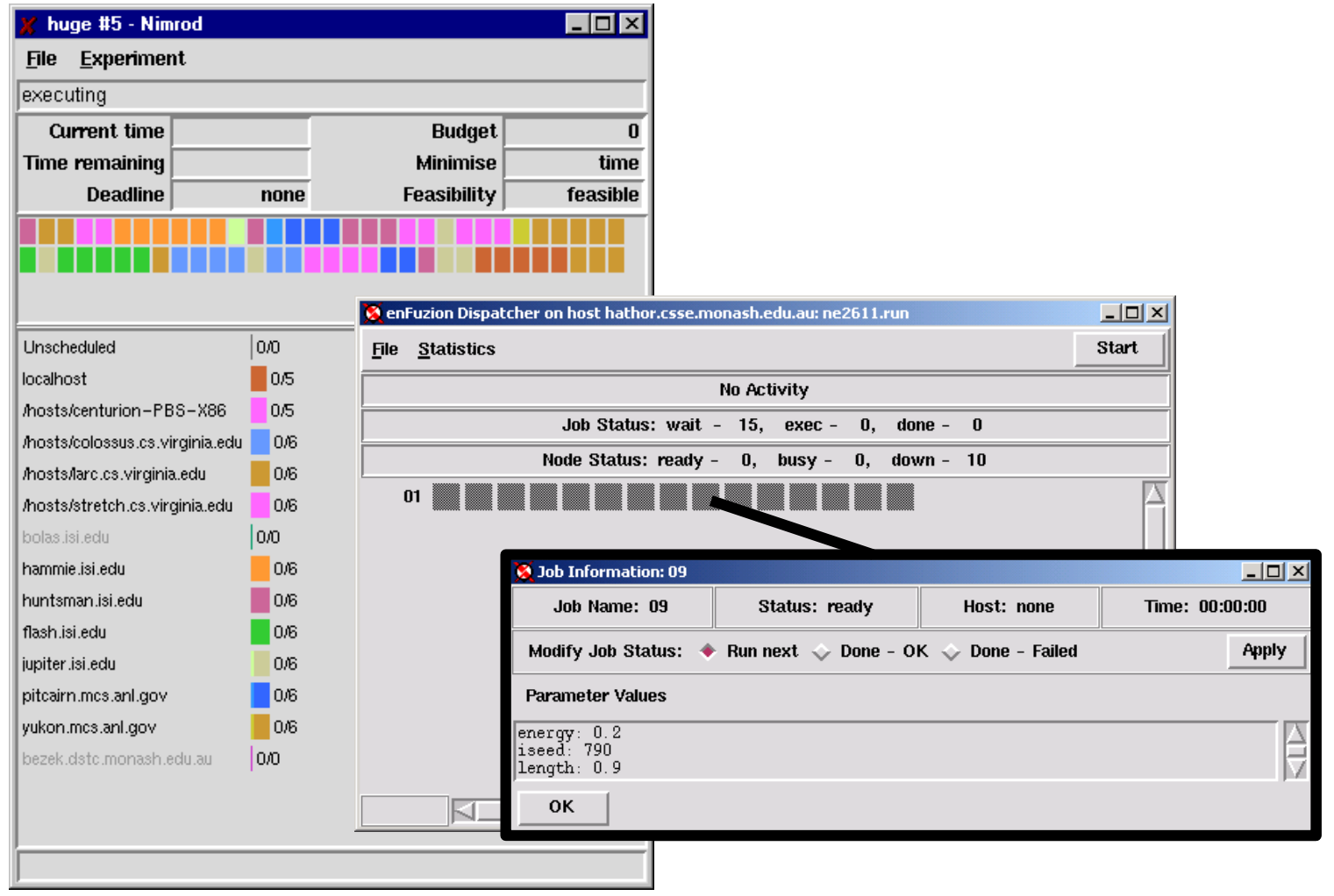

Figure 3 - Nimrod and EnFuzion enginesAn Aerofoil Case Study

Previously, we have applied Nimrod/O to a number of different problems, ranging from air pollution studies, computational electromagnetics and stress analysis [13]. The case study reported in this paper concerns the design of a simple aerofoil. Initially, the problem was developed and solved without Nimrod/O at the Centre for Advanced Numerical Computation in Engineering \& Science (CANCES). Specifically, a simple two dimensional aerofoil was modelled using a FLUENT simulation [19]. The shape of the simulation mesh was generated from the problem input parameters, and FLUENT was used to 
compute the flowfield around the aerofoil, from which lift and drag properties were derived. In addition, a special Fortran program was written to take the solutions from the FLUENT simulation and iteratively search for an optimal wing design using a Simplex method [20]. The aerofoil mesh generated by GAMBIT, had 28089 nodes and 49426 elements, made up of 43090 triangular elements and 6336 quad elements. The convergence criteria for the FLUENT run were set at 1.0e-4. At the time, the experiment was used to investigate the applicability of optimization to the design of an aerofoil, with the goal of maximising the ratio of lift to drag. The solution took some time to develop because it required the development of code specifically tailored to the problem.

The aerofoil shape and configuration is governed by three parameters - the angle of attack, the camber and the thickness, as shown in Figure 4. At a particular configuration of these parameters, the objective function value, the ratio of lift to drag, is maximised. Complete enumeration of the space is infeasible because the number of simulations required is excessive.

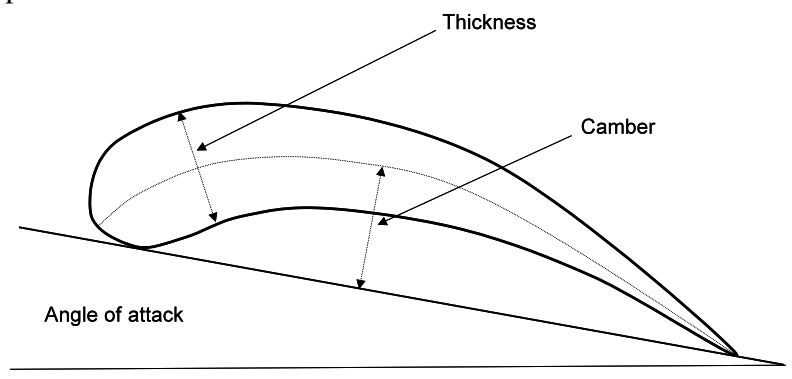

Figure 4 - Aerofoil structure

Following the earlier work performed at CANCES, we applied the Nimrod/O tool to the same problem. Again, the original FLUENT code was used to model the aerofoil, however, rather than using the Fortran code developed specifically for the problem, Nimrod/O was used to perform the search. The study was an outstanding success in three ways:

- It only took about an hour to set up Nimrod/O on the Aerofoil problem.

- The results that were generated by Nimrod/O were better than those of the specific code.

- We were able to explore two algorithms by changing only the plan file

Table 1 summarises the results of the work. The "Best Objective Function" column shows the highest value of the lift/drag ratio. The "Number of function evaluations" is the number of times the FLUENT simulation was executed. These simulations are much more expensive than the optimisation process itself. Therefore, the bulk of the run time is spent in executing the CFD code rather than performing the optimisation calculations. The table shows that our implementation of Simplex and P-BFGS both returned a better result than the tailored Fortran code, and the Nimrod/O Simplex did so with fewer function evaluations. Further, Simplex performed better than P-BFGS, and returned a value of 70.4 with fewer evaluations. The "Wall Clock Time" shows how long the Nimrod/O computations took on the VPAC AlphaServer SC ${ }^{1}$, consisting of Alpha EV68's running at $833 \mathrm{MHz}$. The Tailored Fortran code ran on a different system, so the "Wall Clock Time" is unknown for this method. However, the number of function evaluations for this code is higher than for the Nimrod/O Simplex, and this can be attributed to the different implementations. Further, because the Nimrod/O codes contained some parallelism, they required fewer steps than the sequential Tailored Fortran code. The "Ave number of Procs" column shows the number of processors used on average per step of the algorithm. As expected, Simplex used fewer processors on average than P-BFGS since its internal concurrency is lower.

Figure 4 shows the tracks followed by the Simplex (the black curve) and the P-BFGS (the blue curve) algorithms in the three dimensional space. We have drawn three different iso-surfaces of the objective function, coloured yellow, green and red. The red iso-surface corresponds to an objective function value of

${ }^{1} 32$ Compaq ES40's (4 processors each ) connected by a Quadrics interconnect. 
60 , green corresponds to 52 and the yellow one corresponds to 47 . Thus, in both cases, the tracks show the algorithms iteratively improving the solution until they terminate near the global optimum. Whilst both searches began at similar locations, they followed different paths and converged to different local minima, with Simplex yielding a better result. An interesting observation is that both of the Nimrod/O algorithms that were used yielded good solutions without requiring multiple starting locations. This is presumably due to the relatively smooth nature of the objective function. A movie form of Figure 4 is available on the web at [22].

\begin{tabular}{|l|l|c|c|c|c|c|c|c|}
\hline Method & Algorithm & $\begin{array}{c}\text { Best } \\
\text { Objective } \\
\text { Function }\end{array}$ & $\begin{array}{c}\text { Angle of } \\
\text { Attack }\end{array}$ & Thickness & Camber & $\begin{array}{c}\text { Number } \\
\text { Function } \\
\text { Evaluations }\end{array}$ & $\begin{array}{c}\text { Ave } \\
\text { number } \\
\text { of procs }\end{array}$ & $\begin{array}{c}\text { Wall } \\
\text { Clock } \\
\text { Time } \\
\text { (hh:mm) }\end{array}$ \\
\hline $\begin{array}{l}\text { Tailored } \\
\text { Fortran }\end{array}$ & Simplex & 52.8 & 0.8 & 0.03 & 0.10 & 67 & 1 & Unknown \\
\hline Nimrod/O & Simplex & 70.4 & 1.13 & 0.02 & 0.11 & 41 & 2.7 & $10: 08$ \\
\hline Nimrod/O & P-BFGS & 65.6 & 2.98 & 0.05 & 0.08 & 121 & 6.4 & $17: 13$ \\
\hline
\end{tabular}

Table 1 - Case study results

In this paper we have not discussed the parallel performance of Nimrod/O, as this is the topic of another paper. Table 1 shows that we only managed to utilise a small number of processors for each of the Nimrod/O runs, but since we only considered one run per method, the concurrency is not high. In other studies, we have demonstrated that it is possible to utilise a 64 processor cluster quite effectively by running multiple searches concurrently, yielding both a speedup over an enumerative search running on the same cluster and also running the search procedure on a single processor. More importantly, in this paper, we are concerned with the application of the tool to a new problem, highlighting its ease of use and excellent performance.

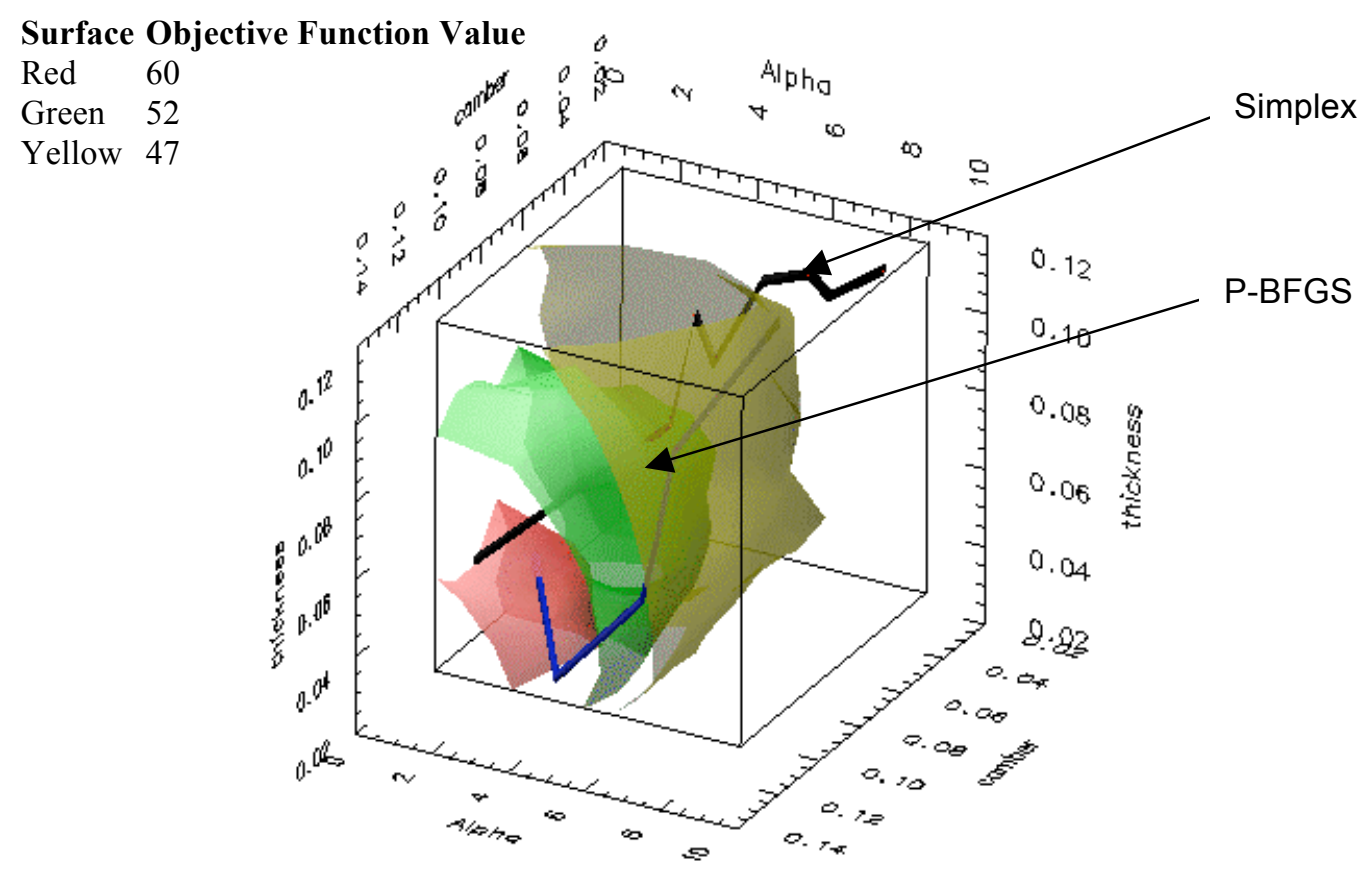

Figure 4 - Simplex and P-BFGS search tracks 


\section{Conclusion}

In this paper we have described a new design tool called Nimrod/O, and have demonstrated its effectiveness on solving a simple, but realistic, CFD problem. In comparison with an existing tailored simplex search, Nimrod/O allowed us to explore two different optimisation algorithms very quickly, and without any code modification. Both of our algorithms out-performed the tailored code, yielding better results in less time. Importantly, the Nimrod/O design philosophy allowed us to perform automatic design without tuning or modifying the existing FLUENT simulation. The most dramatic result of the work was the ease of application - it only took us about an hour to set up Nimrod/O for a new optimisation problem.

Clearly our work is in its early stages - the case study shown here is only a very simple design. In a real world engineering environment, one would expect the computational models to be much more complex, involving multiple simulation techniques. Also, an industrial strength problem would have more parameters. How well our work scales can only be determined by experimentation. However, the early results reported here and in [13] are extremely encouraging.

The choice of back end technology allows us to abstract the exact nature of the computational platforms, and thus the user is free to utilise machines ranging from a single high end workstation through to the computational Grid. We expect to perform some larger design experiments in the area of mechanical durability in the near future.

\section{Acknowledgments}

This work has been supported by the Australian Research Council (ARC), the Victorian Partnership for Advanced Computing (VPAC) and the Centre for Advanced Numerical Computation in Engineering \& Science (CANCES). The programs were run on facilities at CANCES and VPAC. We would like to acknowledge Ivan Mayer for his assistance, and Jimmy Li for his work on the original optimization code.

\section{References}

[1] Abramson D., Sosic R., Giddy J. and Hall B., "Nimrod: A Tool for Performing Parametised Simulations using Distributed Workstations", The 4th IEEE Symposium on High Performance Distributed Computing, Virginia, August 1995.

[2] Abramson, D. "Parametric Modelling: Killer Apps for Linux Clusters", The Linux Journal, \#73, May 2000 , pp $84-91$

[3] Lewis, A., Abramson D., Sosic R., Giddy J., "Tool-based Parameterisation : An Application Perspective", Computational Techniques and Applications Conference, Melbourne, July 1995.

[4] Foster, I. and Kesselman, C. Globus: "The Grid: Blueprint for a Future Computing Infrastructure". Morgan Kaufmann Publishers, 1999.

[5] Eldred, M., Outka, D., Fulcher, C. and Bohnhoff, W. "Optimization of complex mechanics simulations with object-oriented software design.", Proceedings of the $36^{\text {th }}$

IAA/ASME/ASCE/AHS/ASC Structures, Structural Dynamics, and Materials Conference, pp 2406 2415, New Orleans, LA, April 1995

[6] Czyzyk, J, Owen, J. and Wright, S. "Optimization on the Internet", OR/MS Today, October 1997.

[7] Casanova, H. and Dongarra, J. "NetSolve: A Network Server for Solving Computational Science Problems", The International Journal of Supercomputing Applications and High Performance Computing, Vol 11, Number 3, pp 212-223, 1997.

[8] Parker, S. and Johnson, C. "SCIRun: A Scientific Programming Environment for Computational Steering", Proceedings of IEEE Supercomputing 1995, San Diego, December 95. 
[9] McCorquodale, J., Parker, S., Davison de St. Germain, J. and Johnson, C., "The Utah Parallelism Infrastructure: A Performance Evaluation", 2001 High Performance Computing Symposium (HPC'01), Advanced Simulation Technologies Conference, April 22-26, 2001, Seattle, Washington (USA).

[10] Taha, H., (1992) “Operations Research: An introduction” Macmillan Publishing Company, New York, 5th Ed, 822 pages.

[11] Press. W. H., "Numerical Recipes: the Art of Scientific Computing”. Cambridge University Press, 1986.

[12] Gill, P.E., Murray, W. and Wright. M, "Practical Optimization". Academic Press, London, 1981.

[13] Abramson, D, Lewis, A. and Peachy, T., "Nimrod/O: A Tool for Automatic Design Optimization", The 4th International Conference on Algorithms \& Architectures for Parallel Processing (ICA3PP 2000), Hong Kong, 11 - 13 December 2000.

[14] Abramson, D, Lewis, A. and Peachy, T. "Case Studies in Automatic Design Optimisation using the PBFGS Algorithm", 2001 High Performance Computing Symposium (HPC'01), Advanced Simulation Technologies Conference, April 22-26, 2001, pp 104 - 109, Seattle, Washington (USA).

[15] Grimshaw, A. and Wulf, W., et al, "The Legion vision of a woroldwide virtual computer", Communication of the ACM, 40(1):39-45, 1997.

[16] Foster, I., Kesselman, C., Globus: A Metacomputing Infrastructure Toolkit, International Journal of Supercomputer Applications, 11(2):115-128, 1997.

[17] Litzkow, M., Livny, M. and Mutka, M.W., "Condor - A Hunter of Idle Workstations", proceedings of the $8^{\text {th }}$ International Conference of Distributed Computing Systems, pp 104-111, June 1988.

[18] Abramson, D., Giddy, J. and Kotler, L. "High Performance Parametric Modeling with Nimrod/G: Killer Application for the Global Grid?", International Parallel and Distributed Processing Symposium (IPDPS), pp 520- 528, Cancun, Mexico, May 2000.

[19] http://www.fluent.com/

[20] Nelder, J.A., and Mead, R.,"A simplex method for function minimization", Comput. J., 7, pp. 308313, 1965.

[21] Fletcher. R. "Practical Methods of Optimization.” 2nd ed., Wiley, New York, 1987.

[22] http://www.csse.monash.edu.au/ davida/nimrodo/aerofoil.mpg

[23] van Laarhoven, P.,Aarts E., (1987): "Simulated Annealing: Theory and Applications" D Reidel Publishing Company, Dordecht, 186 pages. 